\title{
Diagnostic Investigation of Tune and Tune Shift in the IPNS RCS
}

\author{
J. C. Dooling, F. R. Brumwell, and G. E. McMichael ${ }^{1}$ \\ Argonne National Laboratory
}

\begin{abstract}
The Intense Pulse Neutron Source (IPNS) Rapid Cycling Synchrotron (RCS) accelerates $50 \mathrm{MeV}$ protons to $450 \mathrm{MeV} 30$ times per second for spallation neutron production. Average current from the RCS has recently exceeded $16 \mu \mathrm{A}$ with peak instantaneous current approaching $15 \mathrm{~A}$. The RCS makes efficient use of $21 \mathrm{kV}$ of RF accelerating voltage and uses phase-modulation between the two rf cavities to damp vertical instabilities. Split-ring electrodes in the ring suggest an anomalous tune shift that increases with time in the acceleration cycle. Based on a background gas pressure of $1 \mu$ Torr, the neutralization time for the beam is approximately $0.5 \mathrm{~ms}$ at injection suggesting the beam becomes fully neutralized relatively quickly in the cycle. Over-neutralization of the beam can lead to a positive tune shift that is presumably incoherent. Studies are underway to characterize the ionization within the RCS using the existing Profile and Position System (PAPS) and a newly installed Retarding Field Analyzer (RFA). Also a newly installed fast, deep-memory digitizing oscilloscope allows the entire history of a single acceleration cycle to be recorded from all four components of the split ring electrodes simultaneously at a rate of $250 \mathrm{MS} / \mathrm{s}$.
\end{abstract}

\section{INTRODUCTION}

The Intense Pulsed Neutron Source (IPNS) Rapid Cycling Synchrotron (RCS) accelerates $3 \times 10^{12}$ protons from $50 \mathrm{MeV}$ to $450 \mathrm{MeV}, 30$ times per second. The bare $\mathrm{x}$ and $\mathrm{y}$ tunes are $\mathrm{Q}_{\mathrm{x}} \sim 2.2$ and $\mathrm{Q}_{\mathrm{y}} \sim 2.35$. Observations of spectra recorded from capacitive pickup electrodes within the RCS suggest that for a portion of the beam, the tune may be increasing for part of the accelerator cycle. In 1999, looking at data from these "Pie" position electrodes[i] using a gated spectrum analyzer, a beam loss phenomena referred to as "prespill" was clearly seen to be the result of a resistive wall (vertical) instability as had been previously surmised[ii]. The instability causes a 50 percent loss of beam at the end of the acceleration cycle if left unchecked. The resistive wall instability is controlled through phase modulation (PM) of the rf accelerating voltage. The phase modulator or "scrambler," varies the position of one of the two rf accelerating cavities by sinusoidal amplitude of $100 \mathrm{mrad}\left(6^{\circ}\right)$ near twice the synchrotron frequency. The scrambler circuit also introduces amplitude modulation (AM) at the same frequency. "Slow-Q" (captured charge) signals with and without the scrambler are presented in Fig. 1.

\section{RESISTIVE WALL INSTABILITY}

Since the mid-1980s, the scrambler has been used to stabilize the bunch during the last few milliseconds of the acceleration cycle. Figure 2 shows two spectra taken just prior to extraction, $14 \mathrm{~ms}$ after injection, from a vertical pie electrode using an HP4396B spectrum analyzer (SA). The spectrum presented in Figure $2 \mathrm{a}$ is that with the scrambler modulation on from $9.5 \mathrm{~ms}$ to $11.5 \mathrm{~ms}$, and that in $2 \mathrm{~b}$ is with the scrambler off. Significant side-bands are in evidence in the latter spectrum and are believed to reflect the vertical tune, $\mathrm{Q}_{\mathrm{y}}$. Amplitudes of the sidebands are a strong function of circulating charge.

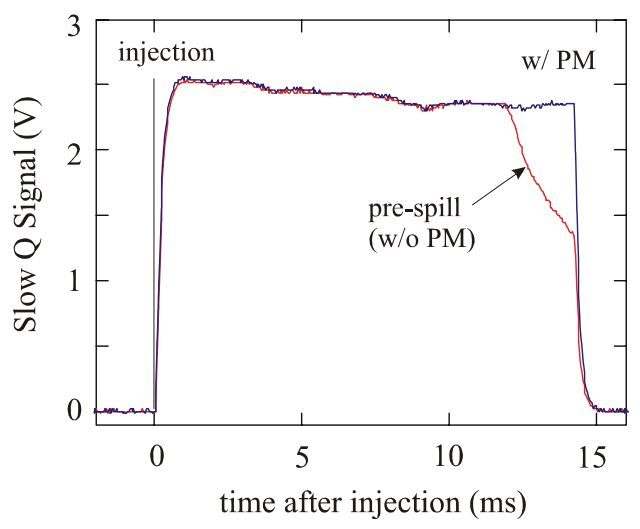

FIGURE 1. Captured RCS charge with and without PM.

The sidebands correspond to $\left(\mathrm{n} \pm v_{\mathrm{y}}\right) \mathrm{f}_{\mathrm{o}}$ for $\mathrm{n}>\mathrm{Q}_{\mathrm{y}}$, in agreement with a resistive wall instability[iii]. The scrambler damps the transverse instability presumably by increasing the energy spread of the bunch. One way this can occur is by establishing a parametric oscillation[iv], where the bunch rotates in longitudinal phase space while maintaining a constant emittance.

${ }^{1}$ The submitted manuscript has been created by the University of Chicago as Operator of Argonne National Laboratory ("Argonne") under Contract No. W-31-109ENG-38 with the U.S. Department of Energy. The U.S. Government retains for itself, and others acting on its behalf, a paid-up, nonexclusive, irrevocable worldwide license in said article to reproduce, prepare derivative works, distribute copies to the public, and perform publicly and display publicly, by or on behalf of the Government. 


\section{FAST RING DATA}

One concern regarding the SA is the degree to which it averages and possibly distorts the actual bunch signal during the cycle. To reduce noise, 10 sweeps are averaged to generate a single spectrum; each spectrum requires approximately 6 minutes to produce at a $\mathrm{BW}$ of $30 \mathrm{kHz}$. Thus at $30 \mathrm{~Hz}$, we are sampling over 10,000 acceleration cycles; i.e., 10,000 bunches. The present generation of fast, deep-memory oscilloscopes makes it possible to produce fast spectra from a single $14 \mathrm{~ms}$ acceleration period. Using a Tektronix TDS7254 oscilloscope with $16 \mathrm{MB}$ of fast memory, the entire RCS cycle can be recorded simultaneously on four channels at a rate of 250 MSamples per second. The penalty for speed is significantly higher noise with respect to the SA due to the greater oscilloscope bandwidth $(2.5 \mathrm{GHz})$.
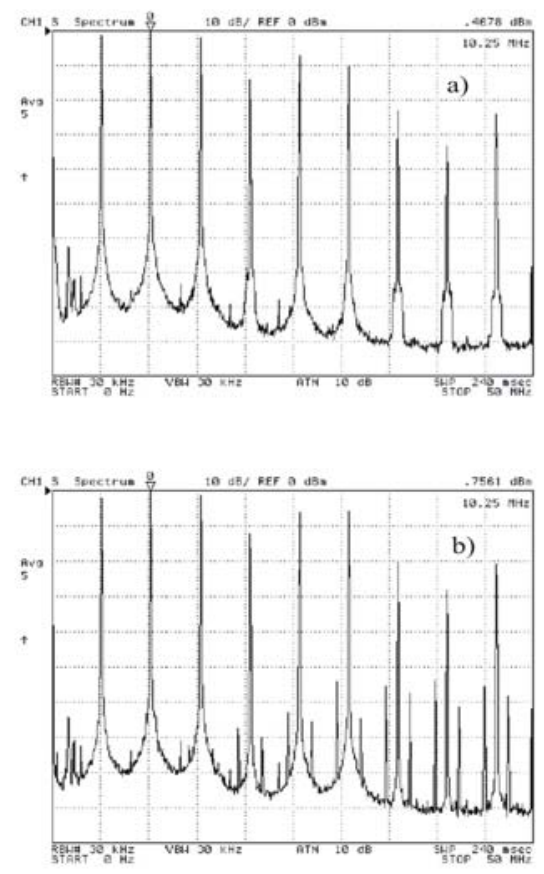

FIGURE 2. Bunch Spectra near extraction a) with scrambler and b) without scrambler.

To evaluate the above concern, a comparison has been made of spectra obtained from both the SA and oscilloscope with the data given in Figure 3. Approximately $0.3 \mathrm{~ms}$ after injection, the top pie electrode signal recorded with the SA exhibits strong sidebands as shown in Figure 3a. The sample period is $0.1 \mathrm{~ms}$. Likewise, sideband structure can also be seen in the spectra generated from TDS7254 oscilloscope data and presented in Figure 3b. The time data was recorded at the same point in the cycle from an adjacent pie electrode during an 80 microsecond period ( $20 \mathrm{kS}$ interpolated to $2^{15}$ values for the FFT). The individual bunch shape recorded with the oscilloscope differs slightly from that of SA but both represent an average. Note that the noise floor is several orders of magnitude higher for the oscilloscope generated spectrum. The noise level in the SA data is typically in the range of $10^{-12} \mathrm{~W}$. Sidebands indicate a vertical fractional tune of 0.28 ; the horizontal tune is 0.20. Irrespective of the noise level, the two techniques do indicate similar spectral features.
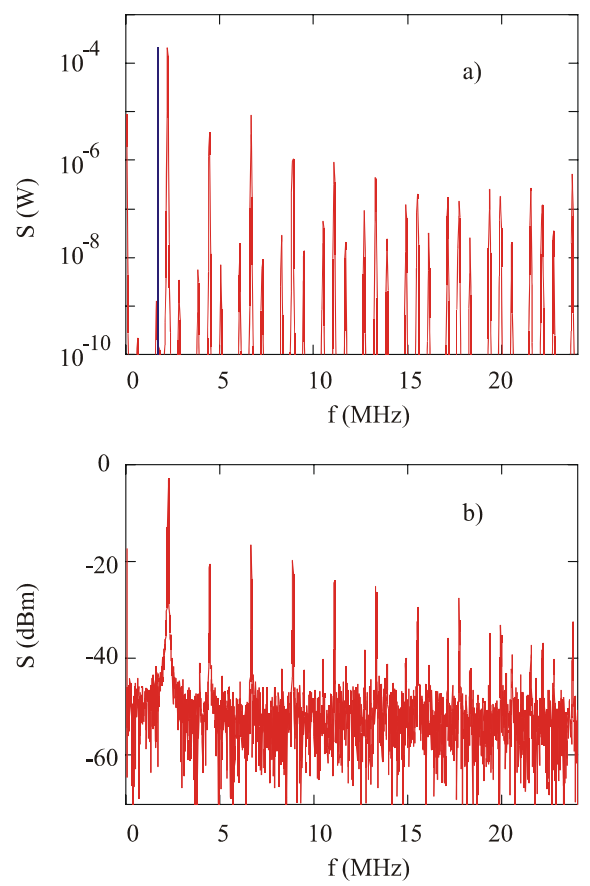

FIGURE 3. Comparison of top pie electrode data recorded with a) SA and b) oscilloscope $0.3 \mathrm{~ms}$ after injection.

\section{SCRAMBLER STUDIES}

The scrambler helps to damp oscillations that are indicative of destructive instabilities; these oscillations can appear as spectral features such as the sideband structure shown in Figure 1b. While the scambler removes one type of narrow band (coherent) structure from the spectra, it introduces other features which are broader in nature. Figure 4 presents pie spectra at two times in the RCS cycle, $10 \mathrm{~ms}$ and $11 \mathrm{~ms}$ after injection. The latter curve has been shift downward two orders of magnitude to make it more visible. The scrambler begins at $9.4 \mathrm{~ms}$ after injection; however, only the primary bunch harmonics are visible at $10 \mathrm{~ms}$. One millisecond later, the spectrum has changed with a pair of broadband, transverse modulation features present on either side of the bunch harmonics. The modulation features are thought to be transverse because they do not appear on longitudinal diagnostics 
such as the beam current toroid (CT) or resistive wall monitor (RWM) in the RCS. Thus, coupling from longitudinal motion into the transverse plane may also help to damp the resistive wall instability. The broad sidebands are not present when the scrambler is off.

Knowing that the scrambler generates sidebands, an effort was made to see if the these spectral features could be observed elsewhere in the acceleration cycle. The scrambler electronics allows for two modulation periods at different times in the cycle. Keeping one at

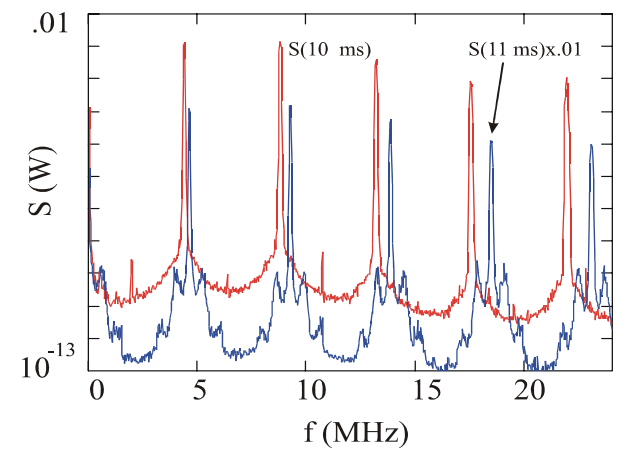

FIGURE 4. Bunch spectra from top pie electrode $10 \mathrm{~ms}$ and $11 \mathrm{~ms}(\mathrm{x} 0.01)$ after injection. Scrambler begins at $9.4 \mathrm{~ms}$.

the normal time for stability while varying the other, it was possible to stimulate the bunch and observe sidebands. The scrambler study was first conducted in December 1999 and repeated again in October 2001. The second test generally repeated the results of the earlier study. Scrambler study measurements are given in Fig. 5. Background gas pressure in the RCS is on the order of $1 \mu$ Torr. Assuming a background of $\mathrm{N}_{2}$; the neutralization time, $\tau_{\mathrm{n}}=0.5 \mathrm{~ms}$ at injection and $1.0 \mathrm{~ms}$ at extraction; therefore, the beam becomes fully neutralized. The tune shift introduced by partially compensated space-charge, may be expressed as[v],

$$
\Delta v(\tau)=-\frac{\mathrm{I}_{\mathrm{p}}(\tau) \mathrm{R}\left(1-\gamma^{2}(\tau) \mathrm{f}_{\mathrm{e}}(\tau)\right)}{\mathrm{I}_{0} \varepsilon_{\mathrm{n}} \beta^{2}(\tau) \gamma^{2}(\tau)}
$$

where $f_{e}$ is the fractional neutralization. If overneutralization is allowed and $\mathrm{f}_{\mathrm{e}}(\mathrm{t})=\mathrm{t} / \tau_{\mathrm{n}}$, where $\mathrm{t}$ is time measured from injection, then the tunes predicted by Eq. 1 are as presented in Fig.5. Some of the structure in the measured tunes may be the result of the pulsed quadrupole magnets in the RCS, the current waveforms of which are given at the bottom of Fig.5.

\section{DISCUSSION AND FURTHER WORK}

Neutralization through ionization means electrons and ions are generated in the background gas, perhaps leading to the formation of a plasma. A plasma sheath forming around the beam would both shield the space charge potential of the beam from the wall as well as carry image current. Plasma formation would be enhanced in the combined-function magnet sections of the RCS which cover just over half of the beam path. Charge confinement times in the magnets should be longer then in the field-free straight sections. Liberated charge is restricted to follow magnetic field lines which lead quickly above and below the beam to a stainless steel liner. Secondary emission from the liner produces more electrons which are again forced to follow field lines back to the beam. An electron
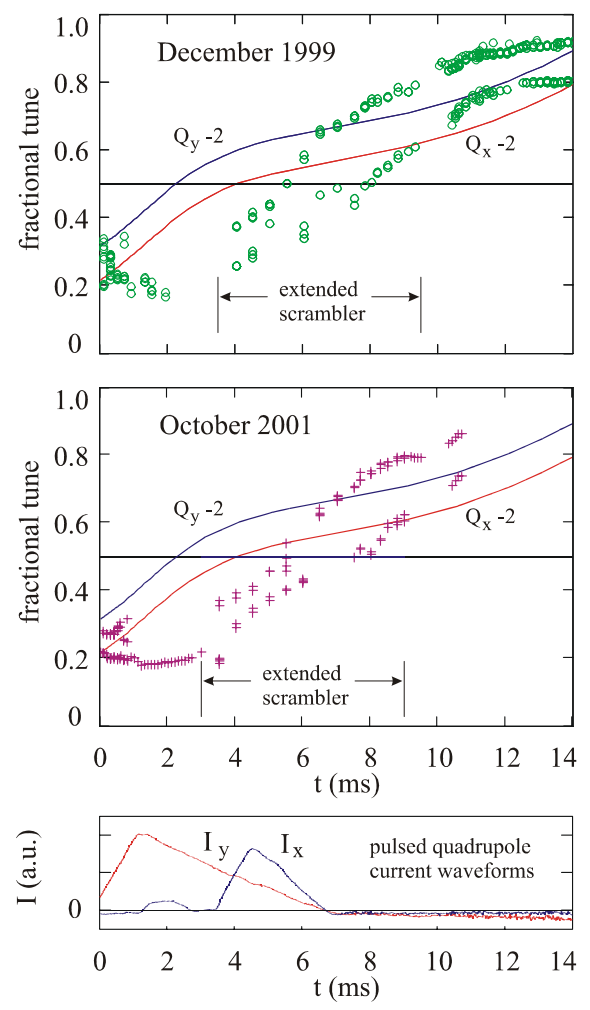

FIGURE 5. Extended scrambler tune measurements

collection grid at the bottom of one straight section, clearly shows the generation of electrons in the beam. We have recently installed a Retarding Field Analyzer (RFA) of the type used in the Proton Storage Ring at LANL and the Advanced Photon Source here at ANL[vi]. With it we hope to learn about the electron density and energy spread near the beam. We look forward to having RFA data during our June run.

\section{ACKNOWLEDGMENTS}

Many thanks to the IPNS Accelerator Operations Group that makes this work possible.

\section{REFERENCES}


i. A. V. Rauchas, et al., IEEE Trans. Nuc. Sci., 28(3), 2338 (1981).

ii. C. Potts, et al., IEEE Trans. Nuc. Sci., 32(5), 3107 (1985).

iii. L. J. Laslett, V. K. Neil, and A. M. Sessler, Rev. Sci. Instrum., 36(4), 1965(436).

iv. M. Bai, et al., Phys. Rev. ST Accel. Beams 3, 064001(2000).

v. M. Reiser, Theory and Design of Charged Particle Beams, Wiley, New York, 1994, p. 274.

vi. R. A. Rosenberg, K. C. Harkay, NIM A, 453, 507(2000) 